\title{
DDOS Attack Prevention on Application Layer
}

\author{
Ashvini P. Pawar \\ BE Computer Engg \\ BVCOE \& RI, Nashik \\ Savitribai Phule Pune \\ University
}

\author{
Kiran S. Kokate \\ BE Computer Engg \\ BVCOE \& RI, Nashik \\ Savitribai Phule Pune University
}

\author{
Anushree P. Sonawane \\ BE Computer Engg \\ BVCOE \& RI, Nashik \\ Savitribai Phule Pune \\ University
}

\author{
Bhagyashree N. Damale \\ BE Computer Engg \\ BVCOE \& RI, Nashik \\ Savitribai Phule Pune \\ University
}

\author{
K.S. Kumavat \\ Asst. Prof. Computer Dept, \\ BVCOE \& RI, Nashik \\ Savitribai Phule Pune University
}

\begin{abstract}
Streathly Denial of Service (DDS) attacks are a complicated threat to the event. Now days, there are an increasing number of DDS attacks against on-line application and Web services. Detecting application layer DDS attack is a hard task. In this, its detection scenario based on the information theory depends on metrics. It has two phases: Behavior monitoring and Detection. In the first phase, the Web user commerce behavior is access from the system log during safe cases. Depends on the observation, Entropy of requests per session and the trust score for each user is evaluated. In the second phase, the suspicious requests are identified depends on the changes in entropy and a rate limiter is identified to downgrade services to malicious attackers. A scheduler is included to planning the session based on the trust score of the user and the system workload.
\end{abstract}

\section{Keywords}

Sophisticated attacks strategy, Low-rate attacks, Intrusion detection, DDS, Application Layer \& Entropy.

\section{INTRODUCTION}

The working systems and network protocols are developed without applying security which results in providing hackers a lot of insecure data on Internet [6]. These insecure and unmatched data are used by DDS attackers as their army to launch attack. An attacker progressively implants attack programs on these unconfident machines. Depending upon complexity in logic of developed programs these executives state are called Handlers or Zombies and are combining called bots and the attack network is called botnet in hacker's collective. Attackers send control instructions to masters, which in turn contact it to zombies for producing attack. To design a system that will detect and prevent the web application from DDS attack [7]. The system will identify whether the user is authorized user or not and then it will detect and prevent the attacks. The attacker will mimic the network traffic pattern of flash event to make the detection tougher. Most of the organized techniques not differentiate the DDS attacks from the surge of legitimate accessing. In distributive denial service attack an area of specification are double check is made before servicing a client, easily deployable, low False rejection rate. Existing technology \& current system technology

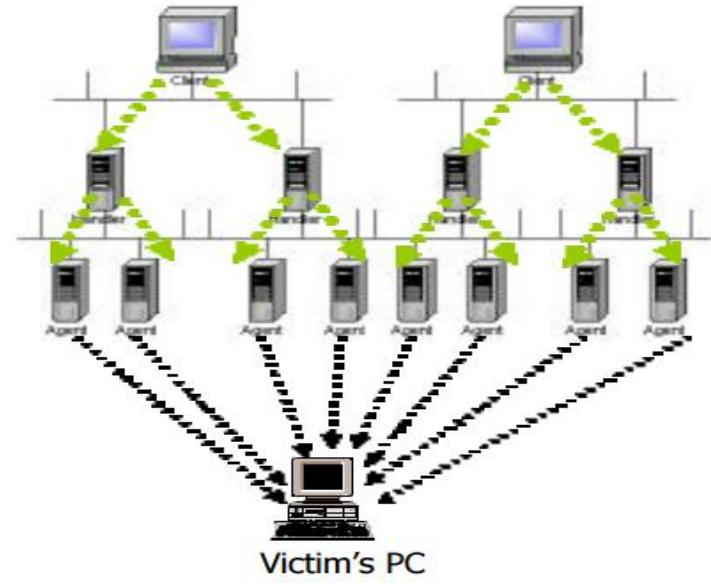

Fig 1: DDoS Architecture

Figure 1 shows normal structure of DDoS attack. DDoS attack is more dangerous because it spread from one to much system and may stop working of whole system.

Existing System:

Traditionally, DDS attacks are carried out at the network layer. Recently, there are an increasing number of DDS attacks against on-line Internet services. These attacks are focus on the application layer. Application layer DDS attacks may focus on wearying the server resources such as Crater, Central Processing Unit, memory, different database bandwidth, and input bandwidth. These attacks are typically more efficient than Transport Control Protocol or User Database Protocol based attacks, requiring less network connections to achieve their bad natured purposes. They are also harder to detect, and they look same to normal starting traffic. Network-layer DDS attacks typically involve a lot of very same traffic; it follows a disk enable pattern, it appears in statistically unexpected quantities, and it looks very different from the surrounding "normal" traffic.

Current System Structure:

Current system firstly count all request then it specify limit for request and set count as per daily use of request. If users want to increase these count then he may specify special request for that. Then validations of user check by using monitoring algorithm, decision algorithm and Entropy algorithm. Entropy algorithms contain rate limiter for deciding limit and scheduler for scheduling purpose. It also consider thermal attacks. It may provide faster execution, also prevent from 
crash, and hang condition. It filters all request in earlier phase hence speed is not affected of the system.

\section{LITERATURE SURVEY}

In the past few years, many institutes have reported a growing number of incidents involving groups of attackers trying to corrupt the systems web related applications by exhausting their resources through distributed denial service (DDS) attacks. Distributed DOS is mainly occurring due to large amount of services are serving in environment and also they can exchanging their data between one another hence there is most chances to occur this attack. For trace back this attack entropy variation method are useful which is work actively for that [1]. Attacker know that preserving application availability is a high priority for most organizations because availability effect of application stipend and therefore any reduction in the quality of service can reduce revenue as well as damage the event's reputation.[2].To protect servers from malicious attacks, a counter-mechanism namely DDS Shield that consists of a suspicion assignment mechanism and a DDSresilient planner. In contrast to prior work, our suspicion mechanism assigns a regular value as opposed to a binary measure to each client lecture, and the scheduler utilizes these values to determine if and when to schedule a session's requests.[3].It uses the security solution and prevent the Internet services Denial services used as changing direction to hide other illegal activities.[4].It separated web object with grouping method .These method used two steps first one is learning and second is detection state.[5].Finding application layer denial attacks are not easy task. It totally depends on the observation task and external environment of request per lecture. These methods are differentiate the attack session with high range detection .DDS attacks involve in saturating the target machine with external communications requests, such that it cannot respond to permissible traffic. Such attacks usually result in a server overload. DDS attacks are implemented purposefully to force the targeted computers to reset, or to consume its resources such as network bandwidth, computing power, and working system data Structures so that it can no longer provide its intended service. To launch a DDS attack, the attackers first build a network of compromised computers that are used to generate the huge volume of traffic needed to oppose services to legitimate users of the victim. Then the attacker installs attack tools on the hosts machine of the attack network. The hosts machine running these attack tools are known as zombies, and they can be used to attack under the control of the attacker. In addition, the attacker will imitate the network traffic pattern of flash event to make the detection tougher. Most of the current techniques cannot discriminate the DDS attacks from the surge of legitimate accessing.

Most of DDOS attack are occur due to distributed system and it may affect all system in network i.e. one system is affected in network by DDoS attack may affects by its communication to other system. It is impossible to completely prevent DDoS attack but it is possible to secure system from maximum affect of DDoS attack [8],[9].

\section{SYSTEM OVERVIEW}

System overview contain system methodology as well as system requirement for developing system as follows-

\subsection{System Methodology}

As shown in figure 2 the current systems and network protocols are developed without applying security engineering which results in providing attackers a lot of inhibited machines on Internet. System Overview shows all description about the work done under the system. In system first of all user Login to system then it request to the server for checking details that user is valid or not. After that desired value and threshold value are checked by server for checking or measuring threshold condition and then decided that user is valid or not. If user is Hacker then access will be denied for that and is not then user will be proceeding. If user is valid but he enter wrong information then after checking validity again the permission for access is given to him.

System Overview also contain what is the requirement for the system for development it may contain software, hardware. Software requirement contain the software use to develop system and the software use to store database of system and its feature.

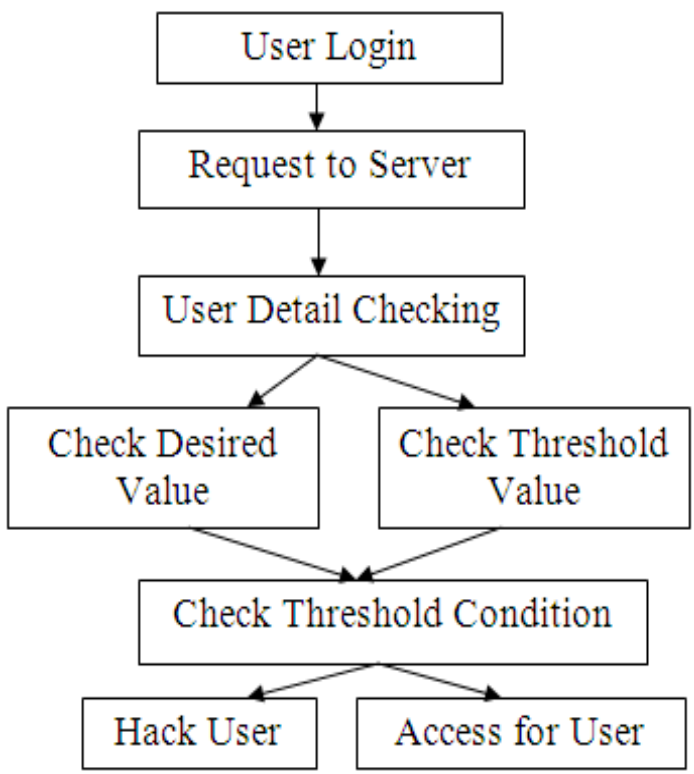

Fig 2: System flow diagram

These inhibited and unmatched machines are used by DDS attackers as their army to launch attack. An attacker gradually inserts attack programs on these inhibited machines. Depending upon complexity in logic of inserted programs these compromised machines are called Masters/Handlers or Zombies and are combining known bots and the attack network is known as bonnet in attacker's community. Attackers send control instructions to masters, which in turn transfer it to zombies for producing attack. In system first user send the request to the server then server checks the client detailed all the request are count then main server finding these request is fake or not. Then final result generated that request is denied or not.

\subsection{System Requirement}

Software Requirements contain operating system as well as languages used for developing entire system-

Operating System: Windows, Linux.

Front End: JAVA

Back End: SQL

System use Java for development due to its feature that is dynamic, object oriented, multithreading, robust and also provide security in implementation. 
System log is past information stored for reference, which can used to find errors. Extract key user browsing features reads the system log and selects some of data from them. Calculate entropy for every user for his every logged in lecture, means it process user data and calculate entropy. Assign trust score calculates trust score for every user. Trust score is average number of user's requests hit per lecture. Variance finding mechanism users requests amount for current lecture will be

Consider in this block, users every request will come here first and will be counted as well. Degree of variance will be calculated here, means with comparison to trust score, how much current requests amount is more or less. Rate limiter is buffer the excess number of request and blocks after some time. Scheduler schedules the buffered requests, means reads requests from buffer and process them after some interval or when load is less on server.

\section{ALGORITHMS}

System supports 3 algorithms for different function as follows:

\subsection{Monitoring Algorithm}

Monitoring algorithm is useful for finding system entropy for time variance as follows:

Input for system log

1. Separated the request arrivals for all meetings page viewing time \& the sequence of input objects for each client from the system $\log$.

2. Compute the entropy of the requests using formula:

$$
H(R)=-\sum_{j} P_{j}\left(r_{j}\right) \log P_{j}\left(r_{j}\right)
$$

\subsection{Detection Algorithm}

Detection algorithm helps system to find out the flow of request coming towards system and also find out malicious request from all coming requests. After finding malicious request system can denied flow of those particular clients.

Input the predefined system of requests per lectures and the belief score for each user.

1. Define the threshold related with the belief score (Tts)

2. Define the threshold for allowable deviation (Td)

3. For each session waiting for detection

4. Extract the requests arrivals

5. Compute the entropy for each lecture using(4)

$$
\mathrm{H}_{\text {new }}(\mathrm{R})=-\sum_{\mathrm{j}} \mathrm{P}_{\mathrm{j}}\left(\mathrm{r}_{\mathrm{j}}\right) \log \mathrm{P}_{\mathrm{j}}\left(\mathrm{r}_{\mathrm{j}}\right)
$$

Compute the degree of deviation:

$$
\mathrm{D}=\left[\mathrm{H}_{\text {new }}(\mathrm{R})\right]-[\mathrm{H}(\mathrm{R})]
$$

6. If the degree of deviation is less than the allowable threshold (Td), and user's belief score is greater than the threshold (Tts), then allow the lecture to get service and the web server Else

The session is malicious; drop it.

\subsection{Entropy Calculation}

Entropy is calculated by using below algorithm

1. Request rij, where $i, j$ I, a set of positive integers.
' $\mathrm{i}$ ' denotes the request no. in session ' $\mathrm{j}$ '.

$|(r j, t)|$ is the no. of requests as $j$, time $t$ then,

$$
\left|\left(r_{j}, t\right)\right|=\sum_{i=1}^{\infty} r_{i j}
$$

2. The changes in the no. of requests $\mathrm{j}$ is given;

$$
N_{j}\left(r_{j}, t+\Delta t\right)=\left|\left(r_{j}, t+\Delta t\right)\right|-\left|\left(r_{j}, t\right)\right|
$$

3. The probability of the requests $\mathrm{j}$, is given bys

$$
\mathrm{P}_{\mathrm{j}}\left(\mathrm{r}_{\mathrm{j}}\right)=\mathrm{N}_{\mathrm{j}}\left(\mathrm{r}_{\mathrm{j}}, \mathrm{t}+\Delta \mathrm{t}\right) / \sum_{i=1}^{\infty} \sum_{j=1}^{\infty} \mathrm{N}_{\mathrm{j}}\left(\mathrm{r}_{\mathrm{j}}, \mathrm{t}+\Delta \mathrm{t}\right)
$$

4. 4. $\mathrm{R}$ be the random variable of the no. of requests during the time $\mathrm{t}$,

$\mathrm{H}(\mathrm{R})=-\sum_{\mathrm{j}} \mathrm{P}_{\mathrm{j}}\left(\mathrm{r}_{\mathrm{j}}\right) \log \mathrm{P}_{\mathrm{j}}\left(\mathrm{r}_{\mathrm{j}}\right)$

5. Based on the characteristics of isolated function, the upper and lower bound of the isolated $\mathrm{H}(\mathrm{R})$

$\mathrm{O} \leq \mathrm{H}(\mathrm{R}) \leq \log \mathrm{N}$

Where, $\mathrm{N}$ is the no. of requests.

Under DDS attack, the no. of request increases significantly \& below equation

$|\mathrm{H}(\mathrm{R})-\mathrm{C}|>$ threshold,

Where, $\mathrm{C}$ is the maximum capacity of session

\section{Rate Limiter}

To reduce false detection, rate limiter is developed. Once the system is calculated by equation compute the degree of variance from the already defined system. The system first sets experiences for acceptable variance. If the computed variance exceeds the experience, then the lecture is forced to eliminate immediately. Otherwise, second level trickle is applied by the rate limiter. The system also defines experience for validating a user based on the trust score. A user is considered to be legitimate only if the trust score exceeds the experiences. Otherwise, the user is considered malicious and the lecture is dropped immediately. The legitimate lectures are then passed to the scheduler for getting service from the server.

\section{Scheduler}

If the user is lawful, then the scheduler schedules the lecture based on the lowest suspicion first policy. The well behaved users will have a little or no variance. In such case, the lawful user gets a faster service. In addition to the scheduling policy, system workload is also considered before scheduling the request for getting service.

\section{CONCLUSION}

In real world internet application is more important and multiple security breakers or attackers are always try to break security of system. Among them most harmful attack is DDOS attack. System includes a strategy to implement stealthy attack patterns, which exhibit a slowly-increasing polymorphic behavior that can evade, or however, greatly delay the techniques proposed in the literature to detect lowrate attacks. Exploiting a vulnerability of the target application, a patient and intelligent attacker can orchestrate sophisticated flows of messages, indistinguishable from 
legitimate service requests. In particular, the attack pattern instead of aiming at making the service unavailable, it aims at exploiting the cloud flexibility, forcing the services to scale up and consume more resources than needed, affecting the cloud customer more on financial aspects than on the service availability. In the future work, system aim on extending the approach to a larger set of application level vulnerabilities, as well as defining a sophisticated method able to detect SIPDAS based attacks in the cloud computing environment.

\section{REFERENCES}

[1] Shui Yu, Wanlei Zhou, Robin Doss, \& Weijia Jia, (2011) "Traceback of DDoS Attacks using EntropyVariations", IEEE Transactions on Parallel and Distributed Systems.

[2] Supranamaya Ranjan, Ram Swaminathan, Mustafa Uysal, Antonio Nucci, \& Edward Knightly, (2009) "DDoS-Shield: DDoS-Resilient Scheduling to Counter Application Layer attacks", IEEE/ACM Transactions on Networking,

[3] Huey-Ing Liu \& Kuo-Chao Chang, (2011) "Defending systems Against Tilt DDoS attacks", $6^{\text {th }}$ International Conference on Telecommunication Systems, Services, and Applications.

[4] Jin Wang, Xiaolong Yang \& Keping Long, (2010) “A New Relative Entropy Based App-DDoS Detection Method", IEEE Symposium On Computers And Communications (Iscc).

[5] S. Yu, W. Zhou \& R. Doss, (2008) "Information theory based detection against network behavior mimicking DDoS attack," IEEE Communications Letters, vol. 12, no. 4, pp. 319-321.

[6] S. Blake, D. Black, M. Carlson, E. Davies, Z. Wang, and W. Weiss. RFC2475: Architecture for Differentiated Services. RFC Editor United States, 1998.

[7] Cisco. "Strategies to Protect Against Distributed Denial of Service Attacks". 17 February 2000. URL: http://www.cisco.com/warp/public/707/newsflash.html (4 Jan. 2002 )

[8] CISCO. "Defining Strategies to Protect Against UDP Diagnostic Port DoS Attacks". September 17, 1996. URL : http://cio.cisco.com/warp/public/707/3.html (4 Jan. 2002)

[9] Raja Azrina Raja Othman "Understanding the Various Types of Denial of Service Attack “.

\section{AUTHOR'S PROFILE}

Ashvini P. Pawar she is Engineering student of computer engineering at Brahma Valley College of Engineering And Research Institute, Nasik under University of Savitribai Phule Pune. Her interest in the field of security.

Anushree P. Sonawane she is student of Engineering student of computer engineering at Brahma Valley College of Engineering And Research Institute, Nasik under University of Savitribai Phule Pune. Her interest in the field of security.

Bhagyashree N. Damale she is student of Engineering student of computer engineering at Brahma Valley College of Engineering And Research Institute, Nasik under University of Savitribai Phule pune. Her interest in the field of security.

Kiran S. Kokate he is student of Engineering student of computer engineering at Brahma Valley College of Engineering And Research Institute, Nasik under University of Savitribai Phule pune. His interest in the field of security.

K. S. Kumavat, ME, BE Computer Engg. Was educated at Pune University. Presently she is working as Head Information Technology Department of Brahma Valley College of Engineering and Research Institute, Nasik, Maharashtra, India. She has presented papers at National and International conferences and also published papers in National and International Journals on various aspects of Computer Engineering and Networks. Her areas of interest include Computer Networks Security and Advance Database. 